\title{
Healthcare costs and outcomes in adult patients with juvenile idiopathic arthritis : a population-based study
}

\author{
Mars, N. J.
}

2019-03-04

Mars , N J , Kerola , A M , Kauppi , M J , Pirinen , M , Elonheimo , O \& Sokka-Isler , T 2019 ,

' Healthcare costs and outcomes in adult patients with juvenile idiopathic arthritis : a population-based study ' , Scandinavian Journal of Rheumatology, vol. 48 , no. 2 , pp.

114-120 . https://doi.org/10.1080/03009742.2018.1475580

http://hdl.handle.net/10138/312751

https://doi.org/10.1080/03009742.2018.1475580

unspecified

acceptedVersion

Downloaded from Helda, University of Helsinki institutional repository.

This is an electronic reprint of the original article.

This reprint may differ from the original in pagination and typographic detail.

Please cite the original version. 


\section{Healthcare Costs and Outcomes \\ in Adult Patients with Juvenile Idiopathic Arthritis: A \\ Population-based Study}

Nina J Mars ${ }^{1,2}$, Anne M Kerola1, 3, Markku J Kauppi ${ }^{3,4}$, Matti Pirinen,2,5,6, Outi Elonheimo ${ }^{1,7}$, Tuulikki Sokka- Isler 8

1 University of Helsinki, Faculty of Medicine, Helsinki, Finland

2 Institute for Molecular Medicine Finland (FIMM), University of Helsinki, Helsinki, Finland

3 Päijät-Häme Central Hospital, Lahti, Finland

4 School of Medicine, University of Tampere, Tampere, Finland

5 Helsinki Institute for Information Technology HIIT and Department of Mathematics and

Statistics, University of Helsinki, Helsinki, Finland

6 Department of Public Health, University of Helsinki, Helsinki, Finland

7 FCG Finnish Consulting Group Ltd, Helsinki, Finland

8 Jyväskylä Central Hospital, Jyväskylä, Finland

Corresponding author:

Nina Mars, MD

University of Helsinki, Faculty of Medicine and Institute for Molecular Medicine Finland

Postal address: Tukholmankatu 8, Biomedicum Helsinki, Helsinki, Finland

Email address: nina.mars@helsinki.fi

Phone number: +358408472156

Abstract: 249 words

Full manuscript: 3240 words 


\section{ABSTRACT}

Objectives: Evidence of the economic burden and long-term outcomes of juvenile idiopathic arthritis (JIA) remain scarce. Our aim was to explore healthcare costs and long-term outcomes in adult patients with JIA.

Methods: We identified all adult patients ( $\geq 18$ years) with JIA who made Jyväskylä Central Hospital rheumatology unit visits between May 2007 and March 2016. We considered individual medians of time-dependent clinical variables. These data were linked to administrative data from the area from the fiscal year 2014, which includes information on all public health care contacts. Healthcare utilization is presented as direct costs in euros $(€)$. Factors affecting direct costs were assessed with a generalized linear model (GLM). Results: In 218 patients, median DAS28-3 was $<2.6$ in $88.6 \%$ in those under age 30 , and in $72.9 \%$ in those over 30 . Median HAQ was $<0.5$ in $85.7 \%$ and $45.4 \%$. In the utilization data (four municipalities, 137 patients) the total annual health services-related direct costs were $432257 €$ (mean = 3 155€/patient/year). Patients using bDMARDs in 2014 for a total of 355 months numbered $36(26.3 \%)$, and the annual cost of bDMARDs was estimated at $355000 €$. Those with active disease had mean costs 2.4 -fold to those with low or no disease activity. A one-point increase in median raw $\mathrm{HAQ}$ incurred an average $228 €$ increase in annual costs $(p=0.03)$.

Conclusions: Most adult patients with JIA seem to manage well with their arthritis, bearing in mind that there still is room for improvement in long-term outcomes. 


\section{INTRODUCTION}

Juvenile idiopathic arthritis (JIA) is a disease entity covering several distinct subtypes sharing chronic arthritis as a common feature, and with symptoms presenting before age 16 (1). Current treatment regimens have led to better outcomes for a medical condition that formerly often inflicted pain and disability (2).

Unexpectedly, little data exist on how patients with JIA are affected by rheumatic inflammation in adulthood. In data mainly from the era before biological disease-modifying anti-rheumatic drugs (bDMARDs), approximately half of all patients with JIA continued in adulthood to have detectable rheumatic inflammation (3).

Active JIA associates with high health care costs in children (4). In the era of biologics, however, evidence of the economic burden remains scarce (5), particularly in adult patients with JIA. The economic burden of rheumatoid arthritis (RA) is actively under study (6-8), but small sample sizes and disease heterogeneity limit research in JIA. Moreover, healthcare utilization in children and adults may differ considerably.

Administrative data have the potential to elaborate knowledge of healthcare resource utilization. We combined a population-based, longitudinal clinical dataset on patients at a rheumatology clinic with administrative data on health services-related direct costs to explore long-term outcome and such costs in adult patients with JIA. 


\section{METHODS}

\section{Patients}

The population-based clinical data cover patients from the Jyväskylä Central Hospital (JCH) area (population 252 000), Finland. Healthcare utilization data were available for four municipalities in this area (population 140000 in 2014). Their primary care is delivered at the local healthcare centres, which rely on $\mathrm{JCH}$ for specialist treatment in rheumatic diseases. The flow diagram is in Figure 1.

\section{Healthcare utilization data}

The healthcare utilization dataset in the fiscal year 2014 came extracted from the electronic medical records (EMR) system for administrative purposes. It is based on routinely recorded information on all public healthcare contacts. A contact is defined as one encounter per diagnosis, for instance, an appointment, one inpatient episode, a phone call, or paperwork that includes logging onto the EMR.

The data comprise contacts with all public healthcare providers in the defined region: physicians and allied health care professionals, including nurses and rehabilitation workers. Both primary and specialty care is covered, including outpatient care, inpatient wards, and the emergency department (ED).

A system similar to diagnosis-related group (DRG), one suitable for both inpatient and outpatient care, served to classify contacts into groups based on recorded diagnoses, either ICPC-2 (International Classification of Primary Care, Second Edition) or ICD-10 (International Classification of Diseases, $10^{\text {th }}$ Revision). The primary diagnosis is recorded, 
and often one or several secondary diagnoses. Our main utilization variable was all-cause, health services-related costs in euros $(€)$ rounded to the nearest integer. This includes costs for both JIA-related and non-JIA-related multimorbidities. Costs are estimated by a costestimation tool owned by Finnish Consulting Group (FGC Ltd). This tool is based on the assumption that costs of similar contacts with specific disease category, age, sex, healthcare unit and provider, and procedures are similar. The estimation tool is trained with a large body of data from both the study area and other municipalities.

To avoid underestimating total costs, we included the costs of contacts without diagnosis codes. In the estimation tool, contacts with no diagnosis code acquire costs similar to the costs of contacts with similar background characteristics.

Only health services-related direct costs were included. In terms of medication costs, we focused only on costs incurred by bDMARDs. Neither social services nor indirect costs were included.

\section{Clinical data}

Patients attending the $\mathrm{JCH}$ rheumatology unit are enrolled prospectively in a structured digital database (GoTreatIT® Rheuma application, DiaGraphIT®) (9), from which we identified all patients with JIA aged $\geq 18$. GoTreatIT monitoring is part of hospital medical records and collects structured data. Data in GoTreatIT can therefore easily be used for register-based research. Because the data are from an adult rheumatology clinic, we were unable to distinguish disease subtypes. 
Clinical data were collected systematically on every visit to the rheumatology unit between May 2007 and March $16^{\text {th }}$ 2016. These data constitute repeated measures, of which we selected a median to avoid the effect of aberrant values, such as high erythrocyte sedimentation rate $(\mathrm{ESR}, \mathrm{mm} / \mathrm{h})$ or C-reactive protein (CRP, $\mathrm{mg} / \mathrm{l})$ due to infections, and to express long-term level of disease activity and functional measures. We assessed disease activity by Disease Activity Score (DAS28-3(ESR)), pain by Visual Analogue Scale (VAS, 0100), and disability by Health Assessment Questionnaire index (HAQ, 0-3). For comparability across rheumatic diseases, DAS28 instead of Juvenile Arthritis Disease Activity Score (JADAS) is recorded. For DAS28-3, the cutoff values chosen were 2.6 and 3.2 , where $\geq 3.2$ represents moderate to high disease activity, $<3.2$ low disease activity, and $<2.6$ minimal disease activity and remission. For HAQ index, we considered 0 to 0.5 as none to mild disability, and above 0.5 as moderate to severe disability.

Age is the age on January 1,2014 . Smoking status was grouped as never and ever smokers (self-reported smoking on at least one visit as of 2007), and medication data as never and ever users of DMARDs, bDMARDs, methotrexate, and oral glucocorticoids (prednisolone, combined with one prednisone user). We used all available information on medication in adulthood. Medication data are routinely collected as of 2007 , but for approximately $10 \%$, medication data (mainly medication use in adulthood) before 2007 has been retrospectively recorded. Costs of bDMARDs in 2014 based on the total months of use were assessed from the clinical data. We estimated the monthly cost to be $1000 € /$ patient (average retail price in 2014). Costs of bDMARDs administered at the hospital rheumatology clinic are by default included in the health services-related costs. The cost of conventional synthetic DMARDs could not be reliably retrieved. 
We selected employment status recorded at the most recent visit to the rheumatology clinic, and categorized it as follows: 1) disabled or pensioner (combined as some had answered "pensioner" although, based on age, they were receiving disability pensions), 2) sick leave or rehabilitation, 3) unemployed, and 4) working or student. We included all causes of sick leave and disability, as they are never distinguishable in our data.

\section{Combining datasets}

In Finland, a register-based study requires no informed consent from study subjects, nor any ethics approval from clinics or hospitals. Approval for the study came from the medical records administration $(\mathrm{JCH})$. We combined the utilization data and clinical data through each patient's unique national identification number.

We studied only adult patients ( $\geq 18$ years on 1 January 2014 , i.e. at the beginning of utilization data collection). From the longitudinal clinical data, we included all visits and measures made in adulthood ( $\geq 16$ years, as the transition age from pediatrics is 16 ).

\section{Statistical analysis}

Our cost data are non-normally distributed and positively skewed. Average-cost analysis included also those with zero costs. For cost comparisons, we used the Wilcoxon rank sum test. For comparing continuous variables, we used the independent t-test when its assumptions were met, and otherwise the Wilcoxon rank sum test. For categorical data, we used Fisher's exact test. In patients with JIA, as disease duration is proportional to age, we assessed only age. We compared patients under 30 with those over 30 , as the latter have 
fallen ill before the era of biologics. We note that possible differences in clinical characteristics between these two groups may also reflect factors other than medication history, such as differing disease durations and general ageing.

A patient was classified as having active disease if making at least one visit to the rheumatology unit with DAS28-3 $\geq 3.2$ in 2014 . We compared the costs of these patients to the costs of those with DAS28-3 $<3.2$ in 2014, which indicates remission or low disease activity.

We assessed factors affecting annual costs in those with non-zero costs with a generalized linear model (GLM) using Gamma distribution and a log link function. Gamma distribution was our choice because it models non-negative data and allows for increasing variance as a function of the mean. We constructed univariate models for age, pain, raw HAQ score, and DAS28-3. All these variables were included in the multivariate analysis, adjusted by sex. No collinearity was detectable. We excluded six cases with costs exceeding the geometric mean by two standard deviations. To increase interpretability, we calculated average marginal effects for variables reaching statistical significance in the multivariate analysis. This displays how the annual costs are affected when a variable increases by one unit, while the other variables remain unchanged.

We also applied a more robust approach by performing a linear regression of inverse normal transformed costs with otherwise a similar model construction as for the GLM but without outlier exclusion. 
No analysis to assess cost differences in those with zero and non-zero costs was performed due to the low number of those with zero costs.

Data handling and statistical analyses were by $R$ version 3.2.4.

\section{RESULTS}

\section{Clinical outcomes}

The clinical data involved $218 \mathrm{JIA}$ patients now adults. Patient characteristics and comparison between the 119 patients younger than 30 and the 99 older than 30 are in Table 1. Average ESR and HAQ were higher in those over $30(p<0.001)$ and also VAS was on average 11.6 points higher in that group $(p<0.001)$. When excluding those over age 60 , results remained unchanged (results not shown).

In the 218 patients, between 2007 and 2016, the mean number of visits to the rheumatology clinic was 9.0 (median 7.0, IQR 3.0-11.0). Those patients with only one visit constituted 10.1\%. The mean observation time was 5.1 years (median 5.6 years, IQR 3.5-6.8), calculated from the first visit to the rheumatology clinic as of 2007, until data extraction in March 2016.

A majority, 90.4\%, had at some point received DMARDs, and $43.5 \%$ had received bDMARDs. In terms of long-term DAS28-3 and HAQ index, proportions per category are in Figure 2. At some point, $18.8 \%$ of patients had smoked. Human leukocyte antigen (HLA) 
B27 was positive for $12.8 \%$ (HLA-B27 status available for $33.5 \%$ ), and $6.4 \%$ were rheumatoid factor-positive (RF, status available for $50.9 \%$ ).

Distribution of employment status between the age groups differed $(p<0.001)$. In those over 30 , those retired or receiving part-time or full-time disability pensions numbered 26 (26.3\%). Of these 26, 20 were under 63, which is currently the youngest regular retirement age in Finland. We may thus assume that at least 20 patients (20.2\%) among those over age 30 received disability pensions. In those over 30 , ones on sick leave or in rehabilitation at the time of their most recent visit to the rheumatology clinic numbered $6(6.1 \%)$.

\section{Healthcare resource utilization}

The administrative data are well-recorded: $82.6 \%$ for diagnosis codes and 97.8 to $100.0 \%$ for all other variables. Of the contacts with no diagnosis code, $71.3 \%$ were other than faceto-face encounters, and $58.4 \%$ non-physician contacts, both of which incur lower costs than, for example, physician contacts.

Healthcare utilization data were available for four municipalities in the area, comprising 137 adult patients with JIA. The total annual health services-related direct costs in these patients were $432257 €($ mean costs = 3 155€/patient/year, median = 1 569€/patient/year). Those with no healthcare contacts during 2014 numbered 17 (12.4\%). Those 81 living outside the area for which we have healthcare utilization data were similar to the 137 with utilization data. Their mean age was 34.3 (31.8 in the 137 with healthcare utilization data), $67.9 \%$ were women (76.6\%), mean DAS28-3 was 1.9 (1.9), mean pain 22.0 (22.4), and mean HAQ index $0.5(0.4)$ 
Cost distributions were similar by sex $(p=0.33)$. Average annual direct healthcare costs were lower $(p<0.001)$ in those 80 patients under age 30 (mean costs $=2386 € /$ year, median $=844 € /$ year), compared with those 57 over 30 (mean costs $=4235 € /$ year, median $=2$ $772 € /$ year).

Those with active disease, defined as at least one visit to the rheumatology unit with DAS28$3 \geq 3.2$ in 2014, numbered 11 (8.0\%). Their mean age was 32.4, mean DAS28-3 in 2014 was 3.7, and mean costs were $6827 € /$ year (median costs $=7$ 076€/year). Their annual costs were higher $(p<0.01)$ than others', i.e. those with DAS28-3 < 3.2 in 2014 indicating remission or low disease activity (mean costs $=2835 € /$ year, median costs $=1311 € /$ year), with their mean health services-related costs being 2.4 times as high. The disease activity parameters, and patient-reported outcomes for those with active disease and for those in remission or with low disease activity are in Table 2.

The 17 patients with zero costs are included in our annual figures; after their omission, results remained unchanged. Specialty care accounted for $80.6 \%$ of total costs, primary care for $14.0 \%$, and the emergency department for $5.4 \%$.

\section{Factors affecting costs}

The only factor associated with costs both in the univariate and multivariate analysis was raw $\mathrm{HAQ}(\beta=0.081, p=0.03$ in the multivariate analysis, marginal effect $=228 €$ for a onepoint increase, $95 \% \mathrm{Cl} 3-453 €$ )(Table 3). A similar association also appeared with the 
inverse normal transformed annual costs (raw $\mathrm{HAQ} \beta=0.089, p=0.001$ in multivariate analysis, $R^{2}=0.25$.

\section{Use of biologic DMARDs}

Of 137 patients in the healthcare utilization dataset, 36 (26.3\%) used bDMARDs in 2014 for a total of 355 months (mean 9.9 months/patient, IQR 8-12 months). The total annual cost of bDMARDs in these patients was $355000 €$, with the mean cost of bDMARDs being 9 $861 € /$ patient/year. The annual health services-related direct costs for the 137 patients of $432257 €$ meant that the annual health services-related direct costs were not far from the annual costs of bDMARDs.

\section{DISCUSSION}

Our main observation was that most patients with JIA have low or no disease activity in adulthood - those with a long-term level of DAS28-3 less than 2.6 at ages under 30 constituted $88.6 \%$ and at those over $30,72.9 \%$. Other studies show in approximately half of adult patients with JIA some detectable inflammation $(3,10)$. One study evaluating patients with disease onset between 1980 and 1985 found that 30 years later, active disease was present in 34\% (11). Our own estimate of those with disease activity was much lower, $8 \%$. That our results show more favourable outcomes compared to previous studies arises from our definition of active disease, which was based on DAS28-3, but they also may to some extent reflect the fact that anti-rheumatic medication is now much more effective than in the 1980s. Our estimate of disease activity may not be directly comparable to others' estimates, because our definition is narrow and confined to a single year. 
Despite seeing a positive trend in terms of disease activity, there still exist patients in need of improved disease control. Almost one in ten had active disease in 2014 . That $12.8 \%$ lacked data for DAS28-3 may indicate that these patients have no symptoms indicative of active JIA.

Between countries, treatment outcomes, as well as costs, vary (12). Due to this heterogeneity and a scarcity of studies on adult patients, costs are not easily comparable. Our mean costs were

$3155 € /$ patient/year, with median costs $1569 €$. We found that during the same fiscal year having an active disease meant that health services-related direct costs more than doubled. In RA, a large share of direct costs result from multimorbidities (13), a pattern likely to be present in our older patients, because multimorbidity is associated with age (14).

As some patients were diagnosed before the $21^{\text {st }}$ century, i.e. before the availability of biologics, we compared that group aged over 30 with those under 30 . The latter presented with better long-term disease control as well as lower patient-reported outcome measures and costs compared to those over 30. Differences in HAQ and ESR are likely explainable by age, and age may also explain why in those over 30 long-term average VAS was higher.

We considered all causes of sick leave and disability pension. In those under 30, disability was rare, but in those older, a disability pension was common, and in $54.6 \%$ the long-term average $\mathrm{HAQ}$ level exceeded 0.5 . Still, most of those over 30 were relatively young, at a mean 44.6. A long history of JIA before the availability of modern, effective diseasemodifying drugs may have permanently affected their joints, reflecting poorly on disability 
measures and healthcare costs. Disability was associated with higher costs: a one-point increase in raw HAQ (individual average level) yielded an average increase of $228 €$ in annual costs.

Current treatment strategies emphasize early and aggressive use of conventional DMARDs, and use of bDMARDs in case of failure of first-line treatment. Medication expenditures represent a substantial part of annual costs, particularly regarding bDMARDs (12), a finding also evident here. Still, the increase in costs may not be as high as expected, and this increase may be advocated, considering the long-term benefits of inflammation alleviation (15). Good disease control may also bring economic benefits, a pattern seen in RA (8). The rheumatology unit in Jyväskylä is highly specialized, and the local healthcare supply chain is elaborate (9), which may positively influence resource utilization, at least in the long run. In Finland, bDMARDs are used when necessary, and nearly half the patients overall had at some point received them. In 2014, a fourth used bDMARDs.

Our clinical data are longitudinal and population-based and include repeated evaluations of patient-reported outcomes. They are constantly updated with false diagnoses removed and corrected, thus increasing accuracy. Previous studies rely mainly on patient- or parentreported estimates for healthcare visits $(4,12)$, and costs are commonly estimated by average unit prices. Our utilization data rely on recordings by healthcare professionals, yielding more reliable data, with every visit leaving its mark in the system. All public healthcare professionals and providers were included, and our costs represent all-cause direct costs, including those of multimorbidities. 
The Finnish healthcare system is mainly public and tax-funded. A private sector exists; it covers some occupational health services and treats some private insurance-holders. In Jyväskylä, rheumatic diseases are treated in public healthcare, but the costs of concurrent diseases may be underestimated.

JIA is a heterogeneous disease: between subtypes, the course of disease, outcomes, and costs vary $(11,16,17)$. Being unable to distinguish among its subtypes is thus a limitation. All JIA subtypes may not be equally represented in our dataset, and those who have reached drug-free remission already in childhood are not likely to be included since their follow-up is primarily in primary care. The use of DAS28 instead of JADAS may underestimate disease activity in JIA. These facts limit the comparability of our results with other outcome studies. Only four patients were lost to follow-up because of moving away before the end of followup, leading to low or no individual costs. Costs are underestimated in terms of conventional DMARDs, analgesics, and medications of co-occuring diseases, which, however, usually incur lower costs than do bDMARDs.

Our goal was to assess health services-related direct costs for this population. Work disability raises the burden on society, as costs due to sick leave, and other indirect costs represent a considerable portion of total costs in adult JIA patients (16). We assume that particularly in those over 30 these indirect costs are substantial.

In conclusion, with current treatment regimens, most patients with JIA seem to do well with their arthritis, bearing in mind that room still exists for improvement in long-term outcomes. Our study suggests that with effective treatment strategies, we may see a positive trend in 
terms of reducing disease activity and disability. Similar studies with even longer follow-up are necessary, and multimorbidity patterns and factors affecting healthcare costs warrant further research.

\section{Funding}

This work was supported by a grant from the Finnish Research Foundation for Rheumatic Diseases.

\section{Acknowledgments}

We thank Santeri Huvinen and Tuuli Pajunen for support in accessing the administrative data and Carolyn Norris for language editing.

\section{Conflicts of interest}

O.E is an employee of the FCG Finnish Consulting Group Ltd, Finland N.M., A.K., M.K., M.P., T.S-I: none declared. 


\section{REFERENCES}

1. Petty RE, Southwood TR, Manners P, Baum J, Glass DN, Goldenberg J et al. International League of Associations for Rheumatology classification of juvenile idiopathic arthritis: second revision, Edmonton, 2001. J Rheumatol 2004;31:390-2.

2. Stoll $\mathrm{ML}$, Cron $\mathrm{RQ}$. Treatment of juvenile idiopathic arthritis: a revolution in care. Pediatr Rheumatol Online J 2014;12:13. eCollection 2014.

3. Minden K. Adult outcomes of patients with juvenile idiopathic arthritis. Horm Res 2009;72 Suppl 1:20-5.

4. Bernatsky S, Duffy C, Malleson P, Feldman DE, St Pierre Y, Clarke AE. Economic impact of juvenile idiopathic arthritis. Arthritis Rheum 2007;57:44-8.

5. Gidman W, Meacock R, Symmons D. The humanistic and economic burden of juvenile idiopathic arthritis in the era of biologic medication. Curr Rheumatol Rep 2015;17:31.

6. Hallert E, Husberg M, Jonsson D, Skogh T. Rheumatoid arthritis is already expensive during the first year of the disease (the Swedish TIRA project). Rheumatology (Oxford) 2004;43:1374-82.

7. Furneri G, Mantovani LG, Belisari A, Mosca M, Cristiani M, Bellelli S et al. Systematic literature review on economic implications and pharmacoeconomic issues of rheumatoid arthritis. Clin Exp Rheumatol 2012;30:S72-84.

8. Barnabe C, Thanh NX, Ohinmaa A, Homik J, Barr SG, Martin L et al. Healthcare service utilisation costs are reduced when rheumatoid arthritis patients achieve sustained remission. Ann Rheum Dis 2013;72:1664-8.

9. Vare P, Nikiphorou E, Hannonen P, Sokka T. Delivering a one-stop, integrated, and patient-centered service for patients with rheumatic diseases. SAGE Open Med 2016;4:2050312116654404.

10. Dimopoulou D, Trachana M, Pratsidou-Gertsi P, Sidiropoulos P, KanakoudiTsakalidou F, Dimitroulas T et al. Predictors and long-term outcome in Greek adults with juvenile idiopathic arthritis: a 17-year continuous follow-up study. Rheumatology (Oxford) 2017;56:1928-38.

11. Selvaag AM, Aulie HA, Lilleby V, Flato B. Disease progression into adulthood and predictors of long-term active disease in juvenile idiopathic arthritis. Ann Rheum Dis 2016;75:190-5. 
12.

Kuhlmann A, Schmidt T, Treskova M, Lopez-Bastida J, Linertova R, Oliva-Moreno J et al. Social/economic costs and health-related quality of life in patients with juvenile idiopathic arthritis in Europe. Eur J Health Econ 2016;17 Suppl 1:79-87.

13. Ohinmaa AE, Thanh NX, Barnabe C, Martin L, Russell AS, Barr SG et al. Canadian estimates of health care utilization costs for rheumatoid arthritis patients with and without therapy with biologic agents. Arthritis Care Res 2014;66:1319-27.

14. Marengoni A, Angleman S, Melis R, Mangialasche F, Karp A, Garmen A et al. Aging with multimorbidity: a systematic review of the literature. Ageing Res Rev 2011;10:430-9.

15. Haapasaari J, Kautiainen HJ, Isomaki HA, Hakala M. Etanercept does not essentially increase the total costs of the treatment of refractory juvenile idiopathic arthritis. $J$ Rheumatol 2004;31:2286-9.

16. Minden K, Niewerth M, Listing J, Biedermann T, Schontube M, Zink A. Burden and cost of illness in patients with juvenile idiopathic arthritis. Ann Rheum Dis 2004;63:836-42.

17. Dimopoulou D, Trachana M, Pratsidou-Gertsi P, Sidiropoulos P, KanakoudiTsakalidou F, Dimitroulas T et al. Predictors and long-term outcome in Greek adults with juvenile idiopathic arthritis: a 17-year continuous follow-up study. Rheumatology (Oxford) 2017;Manuscript in preparation. 


\section{Table 1. Patient characteristics and comparison between patients under and over age}

30. For each, we considered the median of time-dependent clinical variables observed in adulthood (group means of individual medians $\pm S D$ ). For $H A Q$ index, we report also the proportion of those with an individual median of 0 , and the proportion of those with $\mathrm{HAQ}$ index being 0 on the latest rheumatology clinic visit.

\begin{tabular}{|c|c|c|c|c|c|}
\hline & $\begin{array}{l}\text { All } \\
n=218\end{array}$ & $\begin{array}{l}\text { Age }<30 \\
n=119\end{array}$ & $\begin{array}{l}\text { Age } \geq 30 \\
n=99\end{array}$ & $p^{a}$ & $\begin{array}{l}\text { Missing data }{ }^{\mathbf{b}} \\
\%\end{array}$ \\
\hline Age (years) & $32.7 \pm 13.9$ & $22.8 \pm 3.4$ & $44.6 \pm 12.0$ & & 0.0 \\
\hline Women (\%) & 73.4 & 68.9 & 78.8 & $p=0.12$ & 0.0 \\
\hline Body mass index (kg/m2) & $25.0 \pm 5.1$ & $23.5 \pm 4.4$ & $26.7 \pm 5.3$ & $p<0.001$ & 1.8 \\
\hline Ever smokers (\%) & 18.8 & 18.5 & 19.2 & $p \approx 1$ & 0.0 \\
\hline CRP (mg/l) & $4.0 \pm 7.4$ & $2.3 \pm 3.1$ & $6.0 \pm 10.0$ & $p<0.001$ & 9.2 \\
\hline $\operatorname{ESR}(\mathrm{mm} / \mathrm{h})$ & $10.0 \pm 9.4$ & $7.6 \pm 6.2$ & $12.9 \pm 11.7$ & $p<0.001$ & 8.7 \\
\hline DAS28-3 & $1.9 \pm 0.8$ & $1.7 \pm 0.8$ & $2.1 \pm 0.8$ & $p<0.001$ & 12.8 \\
\hline Pain (VAS 0-100) & $22.2 \pm 21.0$ & $17.0 \pm 19.1$ & $28.6 \pm 21.4$ & $p<0.001$ & 0.5 \\
\hline HAQ index (0-3) & $0.4 \pm 0.6$ & $0.2 \pm 0.4$ & $0.7 \pm 0.7$ & $p<0.001$ & 0.9 \\
\hline HAQ index = 0 (\%) & 42.2 & 58.0 & 23.2 & & \\
\hline Latest HAQ index $=0(\%)$ & 48.2 & 64.7 & 28.3 & & \\
\hline Latest SJC28 = $0(\%)$ & 82.4 & 86.0 & 77.9 & & \\
\hline Latest TJC28 = $0(\%)$ & 77.2 & 81.3 & 72.1 & & \\
\hline \multicolumn{6}{|l|}{ Medication in adulthood } \\
\hline Ever DMARDs (\%) & 90.4 & 89.7 & 90.1 & $p \approx 1$ & \\
\hline Ever methotrexate (\%) & 78.0 & 80.4 & 74.1 & $p=0.37$ & 188 \\
\hline Ever prednisone/prednisolone (\%) & 49.7 & 39.2 & 61.7 & $p<0.01$ & 10.0 \\
\hline Ever bDMARDs (\%) & 43.5 & 45.4 & 40.7 & $p=0.55$ & \\
\hline \multicolumn{6}{|l|}{ Employment status c } \\
\hline Disabled or pensioner (\%) & 12.4 & 0.8 & 26.3 & & \\
\hline Sick leave or rehabilitation (\%) & 3.2 & 0.8 & 6.1 & $<0.001$ & 0.0 \\
\hline Unemployed (\%) & 11.9 & 11.8 & 12.1 & $0<0.00$ & 0.0 \\
\hline Working or student (\%) & 72.5 & 86.6 & 55.6 & & \\
\hline
\end{tabular}

a Continuous variables: independent t-test appropriate for BMI. For all other continuous variables, we used the Wilcoxon rank sum test, when assumption of homogeneity of variance was violated Categorical variables: Fisher's exact test

${ }^{\mathrm{b}}$ As we considered medians of time-dependent clinical variables, missing data represents the percentage of unique patients missing data. The majority of missing data for medication is likely explained by patients needing no medication in adulthood. Similarly, those missing data for DAS28-3 are likely to be in remission with no symptoms indicative of active JIA. Of the patients in the healthcare utilization dataset, only $3.6 \%$ (5 out of 137 patients) had missing data for DAS28-3.

${ }^{c}$ At the most recent visit to the rheumatology clinic. Includes also non-rheumatological causes of sick leave and disability. "Disabled" includes part-time disability pension. 
ESR $=$ erythrocyte sedimentation rate, CRP $=$ C-reactive protein, VAS $=$ Visual Analogue Scale, bDMARDs $=$ biologic diseasemodifying antirheumatic drugs, HAQ = Health Assessment Questionnaire, TJC28 = Tender 28-Joint Count, SJC28 = Swollen 28Joint Count

Table 2. Disease characteristics for those with active disease (at least one visit with DAS28$3 \geq 3.2$ in 2014) and those in remission or with low disease activity (DAS28-3<3.2 in 2014). We considered the median of time-dependent clinical variables observed in adulthood (group means of individual medians $\pm S D$ ).

\begin{tabular}{l|l|l|} 
& At least one DAS28-3 $\geq 3.2$ in 2014 & DAS28-3<3.2 in 2014 \\
\hline Number of patients & 11 & 126 \\
DAS28-3 & $3.1 \pm 0.7$ & $1.8 \pm 0.7$ \\
Pain (VAS 0-100) & $30.1 \pm 18.0$ & $21.7 \pm 19.4$ \\
HAQ index (0-3) & $0.9 \pm 0.9$ & $0.3 \pm 0.5$ \\
HAQ index = 0 (\%) & 18.2 & 46.8 \\
Ever DMARDs (\%) & 100.0 & 100.0 \\
Ever bDMARDs (\%) & 81.8 & 50.0
\end{tabular}


Table 3. Factors affecting annual health services-related direct costs. These were assessed in those with non-zero costs by univariate and multivariate regression models. For non-transformed costs we applied a generalized linear model (GLM) with Gamma distribution and a log link function. We also applied a more robust approach by performing linear regression on inverse normal transformed costs.

\begin{tabular}{|c|c|c|c|c|c|c|c|c|}
\hline & \multicolumn{4}{|c|}{ Costs not transformed } & \multicolumn{4}{|c|}{ Costs transformed } \\
\hline & \multicolumn{2}{|c|}{ Univariate } & \multicolumn{2}{|c|}{ Multivariate } & \multicolumn{2}{|c|}{ Univariate } & \multicolumn{2}{|c|}{ Multivariate } \\
\hline & $\beta$ & $\mathrm{p}$ & $\beta$ & $\mathrm{p}$ & $\beta$ & $\mathrm{p}$ & $\beta$ & $\mathrm{p}$ \\
\hline Age & 0.014 & 0.08 & -0.005 & 0.62 & 0.023 & $<0.001$ & 0.001 & 0.94 \\
\hline Sex & & & 0.074 & 0.77 & & & -0.019 & 0.93 \\
\hline Pain & 0.014 & 0.01 & 0.009 & 0.18 & 0.020 & $<0.001$ & 0.007 & 0.17 \\
\hline Raw & & & & & & & & \\
\hline $\mathrm{HAQ}$ & 0.084 & 0.001 & 0.081 & 0.03 & 0.107 & $<0.001$ & 0.089 & 0.001 \\
\hline DAS28-3 & 0.080 & 0.53 & -0.087 & 0.56 & 0.263 & 0.02 & 0.009 & 0.94 \\
\hline
\end{tabular}

$\mathrm{HAQ}=$ Health Assessment Questionnaire, DAS28 = Disease Activity Score 28 
Figure 1. Flow diagram.

Analysis of clinical outcomes

Analysis of healthcare resource utilization

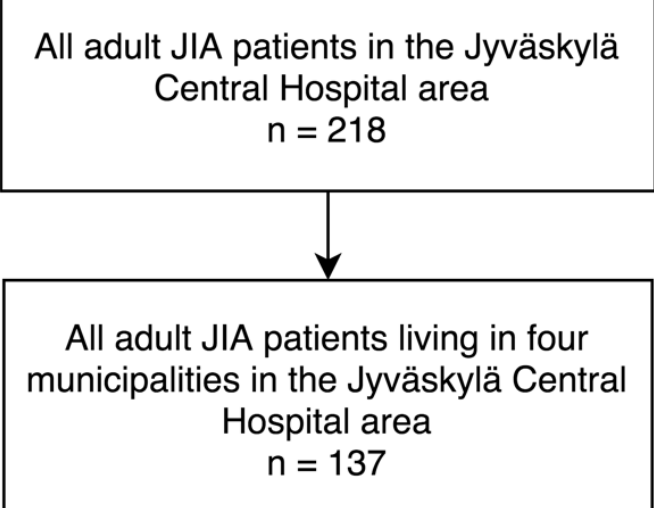

Figure 2. Proportions of long-term DAS28-3 and HAQ disability index (medians of individual repeated measures) in those 119 under and 99 over age 30. DAS28-3 median is based on average 4.8 visits to a rheumatology clinic in those with long-term DAS28-3 $<2.6$, average 5.4 visits in the group 2.6-3.2, and average 8.4 visits in the group $\geq 3.2$.

\section{DAS28-3}

$<2.6-2.6-3.2 \square \geq 3.2$

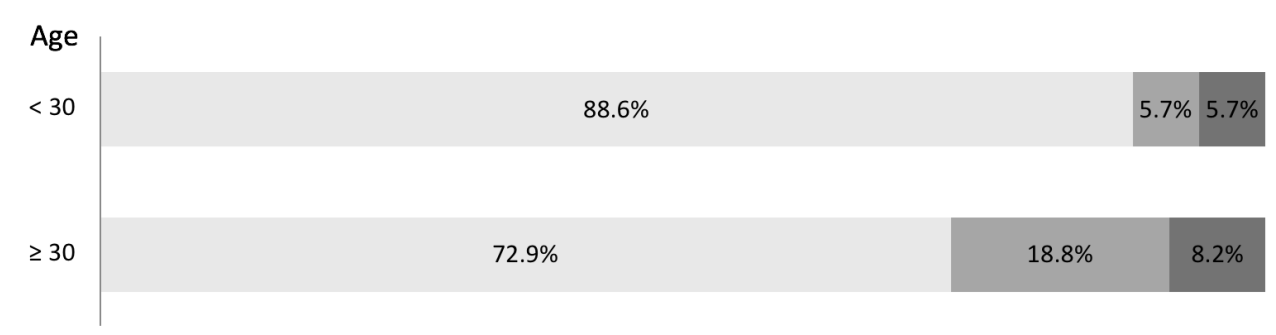

\section{HAQ disability index}

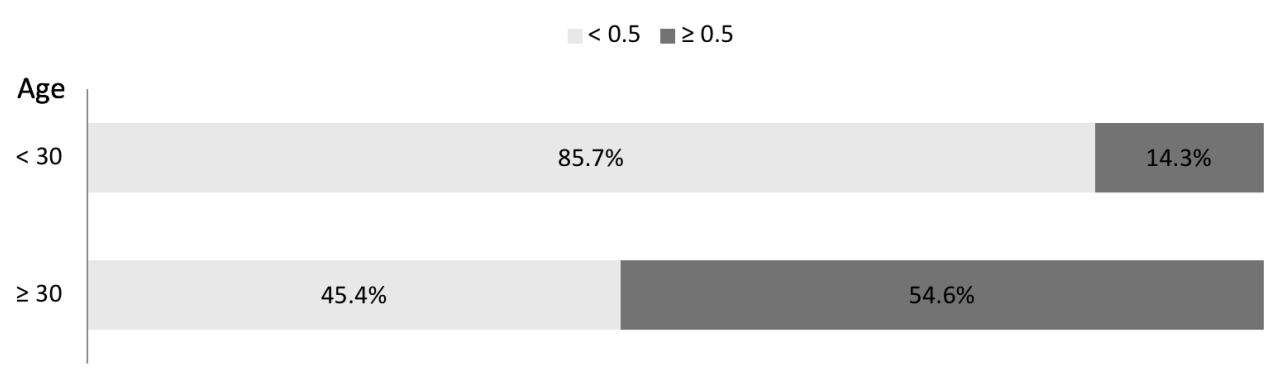

